\title{
Grado de apropiación de los objetos matemáticas en juego en la resolución de problemas. Un ejemplo habitual sobre la determinación de máximos y mínimos
}

\author{
Degree of appropriation about mathematical objects involved in the \\ solving problem: a daily life examples respect to maximum and \\ minimum
}

\author{
Ismenia Guzmán Retamal ${ }^{1}$ \\ iguzmanr@vtr.net \\ Neumarino Rodtriguez Ventura ${ }^{2}$ \\ nrodrig@uvm.cl
}

\begin{abstract}
Resumen
En este artículo analizamos respuestas de estudiantes universitarios de primer año a un problema, en el cual aparece la función $\mathrm{f}(\mathrm{x})=\mathrm{x}^{3}$ usual en textos y cursos de cálculo diferencial. El enunciado lo tomamos del trabajo de Trigueros y Escandón (2008), debido a lo no rutinario del mismo. Un estudio cuantitativo previo de este problema planteado a 90 estudiantes universitarios, arrojó un gran número de respuestas deficientes (Guzmán et al (2010). Por ello decidimos seleccionar algunas respuestas para profundizar su estudio, considerando la teoría de registros de representación de Raymond Duval. El análisis ha dejado en evidencia que los estudiantes no consideran todos los datos del problema y tampoco coordinan los conocimientos representados en los distintos registros de representación: registro simbólico, registro gráfico, de tabla y del lenguaje natural. Según Duval es necesario movilizar la operación de Coordinación entre registros para dejar en evidencia la apropiación de los conocimientos requeridos para trabajar en la solución de un problema.
\end{abstract}

Palabras clave: Cálculo Diferencial. Enunciado no rutinario. Representación semiótica. Teoría de Registros de representación semiótica. Operación de coordinación. Grado de comprensión. Apropiación. Máximos. Mínimos.

\begin{abstract}
In this article we analyzes the responses of university students of first-year facing a problem, which appears the usual function $\mathrm{f}(\mathrm{x})=\mathrm{x}^{3}$ in the calculus texts.. This problem we take of the Trigueros and Escandon (2008) article due to it is not routine problem. A quantitative study about this problem applied to 90 students has revealed that a large number of responses were very weak of the mathematical perspective (Guzman et al (2010). Therefore we decided to select some answers and to depth in this study according register semiotic representation theory of Raymond Duval. The analysis has revealed that students did not consider all the data of the problem, they apply the procedure learned mechanically,
\end{abstract}

\footnotetext{
${ }^{1}$ Académiva Universidad de Los Lagos sede Santiago.

${ }^{2}$ Universidad Viña del Mar.
} 
without coordinating different possible representation registers involved in the solution of the problem: symbolic, graphic, variations tables, and natural language. According Duval is necessary to mobilize the operation of coordination between at least two registers by to put clear the appropriation of the knowledge required to work solving problem. As a result we thought it would need to look more closely at the emphasis on teaching of mathematical objects to go beyond the operative techniques.

Keywords: Differential Calculus. Non routine problem. Theory of semiotic registers of representation. Operation of coordination. Degree of understanding. Appropriation. Maxima. Minima.

\section{Introduccion}

En este artículo mediante un estudio de casos analizamos las dificultades que enfrentan algunos estudiantes de primer año universitarios sobre un problema referido a la determinación de valores extremos de una función real. El análisis sobre la comprensión lo hacemos desde la perspectiva cognitiva de la teoría de registros semióticos de R. Duval. El problema considerado es el siguiente:

Considera la función $\mathrm{h}(\mathrm{x})=\mathrm{x}^{3}$, sabemos que $\mathrm{h}^{\prime}(\mathrm{x})=0$ si $\mathrm{x}=0$, ¿podemos con esto asegurar que en $\mathrm{x}=0$ existe un máximo o un mínimo?

Como se puede apreciar el problema es conocido en la mayoría de los cursos de Cálculo y su bibliografía y se sitúa en el marco de las derivadas, en particular en la problemática de la determinación de máximos o mínimos. Naturalmente se presenta en el registro verbal empleando notaciones simbólicas de los objetos del marco matemático señalado.

En el análisis a priori del enunciado consideramos dos estrategias de resolución.

La primera, situada en el registro gráfico, donde la expresión $h(x)=x^{3}$ se asocia a la representación gráfica de la función, entonces si se conoce la curva de la función h, la respuesta a la pregunta por visualización resulta inmediata, no hay máximo ni mínimo.

Si no se conoce la gráfica de la función entonces se trata de construir la curva con la técnica del "Análisis de curva"3 (trabajo en el registro algebraico-simbólico). Con esas informaciones se construye la tabla de signos de la primera derivada, determinando que la función h es creciente (se puede visualizar el comportamiento de la curva en el registro gráfico) y en consecuencia se responde la pregunta.

\footnotetext{
${ }^{3}$ El análisis de curva contempla los siguientes pasos: Determinar dominio de la función h, Calcular la primera derivada y determinar los puntos críticos de h, Aplicar el criterio de la primera derivada.
} 
La segunda estrategia se sitúa en el registro algebraico-simbólico, donde se calcula h' y se aplica el criterio de la primera derivada (análisis de signos). Luego se verifica que $h^{\prime}(x)>0$ para todo $x$ distinto de cero (de la expresión $3 x^{2}$ ), relacionando este resultado con el crecimiento de la función h, en todo el dominio y también se puede visualizar el comportamiento de la curva en el registro gráfico; en consecuencia se concluye que no existe ni máximo ni mínimo en $\mathrm{x}=0$.

\section{Antecedentes}

Entre las investigaciones afines a la nuestra encontramos la de (Moreno y Cuevas, 2004) señala que el aprendizaje de la matemática en particular el cálculo diferencial, en lo que respecta a determinar máximos o mínimos de una función por parte de los estudiantes está fuertemente influenciada por su enseñanza rutinaria $\mathrm{y}$ descontextualizada, en la investigación se establece que los alumnos aplican los algoritmos de manera mecánica llegando a soluciones inverosímiles.

Esta característica ha perdurado desde los inicios de este tema, puesto que en el siglo XVII, Fermat desarrolló el primer método general para la determinación de máximos y mínimos.

Algunas de las dificultades registradas en las producciones de los alumnos han sido señaladas por Artigue (1998), por ejemplo aquellas ligadas a la complejidad matemática de los objetos básicos de este campo conceptual; en especial los números reales y las funciones entre otras; también dificultades ligadas a la necesaria ruptura con modos característicos de pensamiento algebraico.

El estudio que se refieres a voces de estudiantes (Guzmán, 1998), revela que las respuestas que ellos dan en relaciones con funciones están dadas en un solo registro de representación, ellas se sitúan en el registro en que está dada la pregunta o recurren al registro algebraico que con frecuencias es privilegiado en la enseñanza, sin considerar otras representaciones semióticas.

\section{Marco teorico}

Raymond Duval ha estudiado las representaciones semióticas y su rol en la adquisición de conocimientos matemáticos. Enfatiza que la semiosis es inseparable de una 
diversidad de signos disponibles y que este fenómeno fue reconocido por Pierce en 1931 y ha distinguido tres tipos de signos: los íconos, símbolos e índices Esta clasificación está basada sobre la lógica y ha contribuido a fundar la semiótica. Señala además que Beneviste plantea que la lengua natural es la "organización semiótica por excelencia" y trata de precisar la noción de "sistema semiológico". Pero los problemas de conversión de representaciones de un sistema a otro fueron explicitados mucho más tarde por Chomsky en 1971 y por la reflexión post Saussurienne de Beneviste (1974).

Pero según Duval el análisis de estos autores se limita a la comparación de sistemas semióticos con la lengua natural, a través de producciones culturales independientes entre sí: lengua y música, lengua y arte pictórico, pero no se interesaron en el rol de la diversidad de los sistemas semióticos en el funcionamiento del pensamiento ni en la complejidad de la conversión de las representaciones de un sistema a otro.

La relación entre Representación y Conocimiento es la que Duval estudiará, para lo cual define la noción de Registro de representación Semiótica, más exigente que la de un sistema semiótico. En efecto, un sistema semiótico es un conjunto de signos bien definidos y organizados, con reglas propias de formación. Sin embargo, no todos los sistemas semióticos son registros de representación semiótica. Por ejemplo: los códigos del tránsito no forman un registro, ni el alfabeto Morse.

Un Registro Semiótico es un sistema semiótico que cumple con las tres actividades cognitivas siguientes:

$1^{\circ}$ Constituir una traza o un ensamblaje de trazas de modo que sean identificables como una representación de alguna cosa en un sistema determinado.

$2^{\circ}$ Tratamiento: Consiste en transformar las representaciones al interior del sistema, por sus reglas propias para obtener otras representaciones que aporten nuevos elementos

$3^{\circ}$ Conversión: Consiste en transformar la representación producida en un sistema, en otra representación pero en otro sistema, de modo que se pueda explicitar otras significaciones.

El análisis de los conocimientos y de los obstáculos a los aprendizajes fundamentales, confronta tres fenómenos estrechamente ligados. El primero, es la diversidad de registros, que deja en evidencia que el lenguaje natural es distinto de los lenguajes formales, también, las figuras geométricas son distintas de los gráficos cartesianos. 
Se consideran registros auxiliares las tablas de doble entrada, los esquemas, los sintagmas, las ilustraciones, los cuales son representaciones muy diferentes entre sí y cada uno plantea cuestiones de aprendizajes específicas.

El segundo fenómeno se refiere a la importancia de diferenciar la representación y el contenido representado, esta diferenciación está asociada a la comprensión de lo que una representación representa. Cabe señalar que tal diferenciación no es espontánea y jamás es adquirida en un paso, cualquiera sea el registro y cualquiera sea el nivel de desarrollo del sujeto. El tercer fenómeno es el de coordinación entre diferentes registros de representación, para lo cual es necesario reconocer índices en las representaciones de los registros movilizados para ponerlos en correspondencia. La habilidad de coordinar los registros en juego es esencial para la comprensión de los objetos matemáticos en juego.

Un obstáculo para poner en juego en forma espontánea esta coordinación es la existencia de fenómenos de no congruencia entre las representaciones producidas en registros diferentes. Afirma Duval (2006, p. 159)

"La comprensión no significa dar un salto desde el contenido de una representación hasta el concepto puramente matemático representado sino en relacionar diversos contenidos de representación del mismo concepto. No existe una "mediación semiótica" homogénea sino varias que tienen poco o nada en común. Y como ya hemos visto los contenidos de representación dependen no sólo de lo que es representado sino también de los sistemas de representación usados. Por eso la comprensión matemática requiere una coordinación interna entre los diversos sistemas de representación semióticos posibles que se pueden elegir y usar (Duval, 2000). Sin desarrollar esta coordinación interna los estudiantes no pueden cruzar el umbral de la conversión de representación. La habilidad para movilizar diversas representaciones conjuntamente de manera interactiva o en paralelo depende del desarrollo de esta coordinación, y la comprensión conceptual no es la condición de tal coordinación sino que surge de su desarrollo. En otras palabras, lo que primero importa para la enseñanza de las matemáticas no es la elección del mejor sistema de representación sino lograr que los estudiantes sean capaces de relacionar de diferentes maneras de representar los contenidos matemáticos". 


\section{Elección del problema}

El problema ha sido elegido por dos razones, una de carácter matemático y la otra de carácter didáctico. Desde el punto de vista matemático es un problema usual en cualquier curso de Cálculo, para el cual existen varias estrategias de solución. Desde el punto de vista didáctico, la formulación del enunciado enfrenta a los estudiantes a una pregunta que exige una respuesta que sea justificada con razones matemáticas.

\section{Metodología y análisis de producciones}

Hemos considerado cinco respuestas en las que aparecieron al menos dos registros involucrados y resultan mayoritarias del comportamiento de gran parte de los encuestados. Hemos dado nombres ficticios a los autores de las respuestas.

Partimos por describir el desarrollo de la respuesta, desde el punto de vista matemático y luego analizamos según el marco teórico, los registros movilizados en el desarrollo de las respuestas y la posible coordinación entre ellos. De acuerdo a Duval el grado de comprensión de los objetos matemáticos en juego está dado por la movilización conjunta de los registros involucrados.

Recordemos el problema planteado

Considera la función $h(x)=x^{3}$, sabemos que $h^{\prime}(x)=0$ si $x=0$, ¿podemos con esto asegurar que en $\mathrm{x}=0$ existe un máximo o un mínimo? 
Figura 1 - Producción de Jazmín

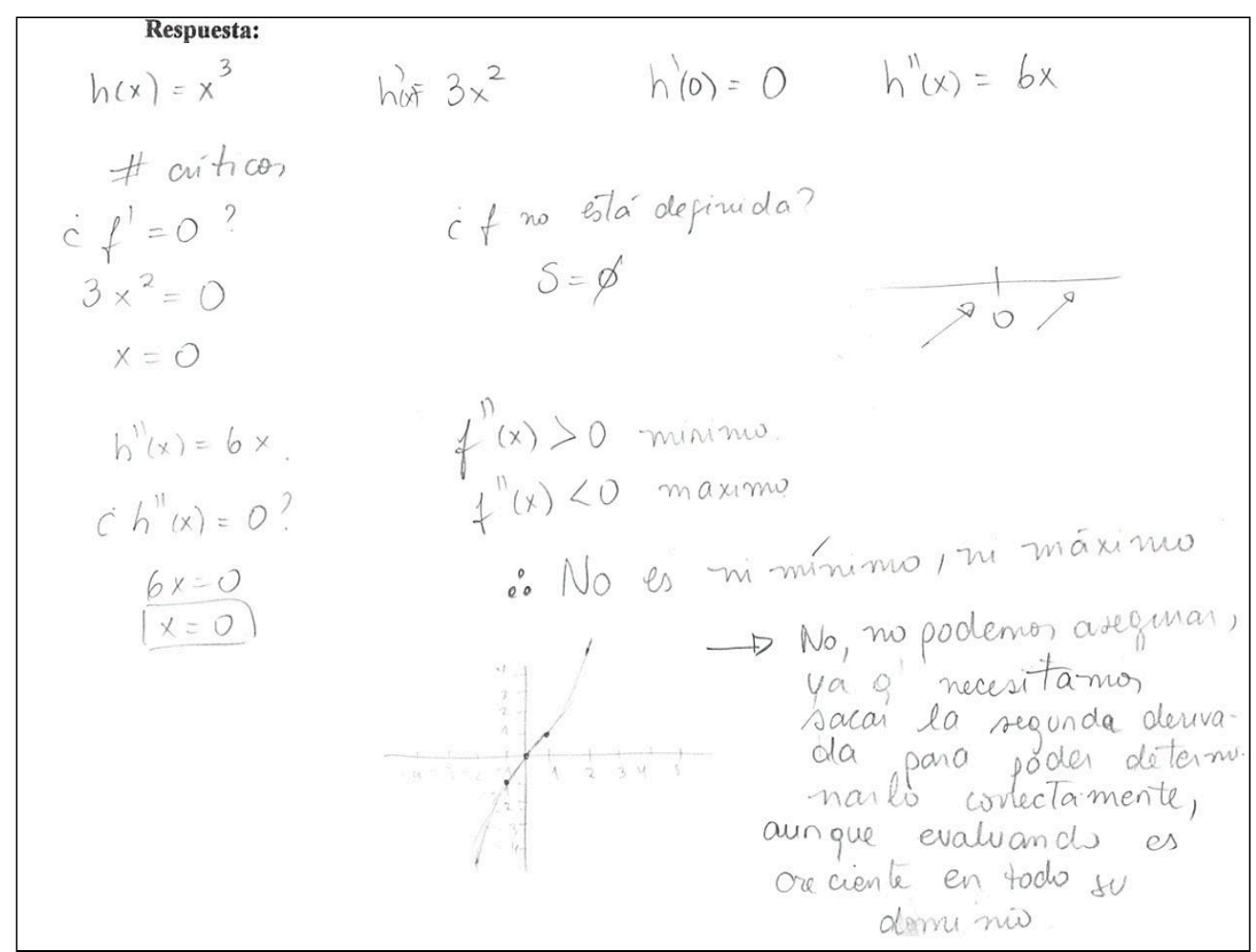

En la primera línea del desarrollo observamos que no toma en cuenta las hipótesis del problema; calcula la primera derivada de h en cero y continúa calculando la segunda derivada . En la segunda línea sigue el procedimiento clásico (del curso) para hallar los puntos críticos. Así verifica la hipótesis. Todo este trabajo está situado en el registro algebraico-simbólico.

El esquema que aparece a la derecha, hace pensar que es el análisis de signos de la función primera derivada, $3 \mathrm{x}^{2}$. Hasta este momento ha movilizado dos registros: el algebraico simbólico y el auxiliar del esquema. Pero no los coordina, puesto que no conecta el significado de las flechas con el crecimiento de la función, dado por el signo de su primera derivada. De haber hecho tal conexión hubiera podido responder directamente que no se puede asegurar que existe máximo o mínimo. Sin embargo, recurre al criterio de la segunda derivada y concluye erróneamente que $\mathrm{x}=0$,

“ no es mínimo ni máximo”. Ya que si la segunda derivada es cero, la función en cero puede tener, un máximo, un mínimo o un punto de inflexión. 
Nota: Cuando la segunda derivada resulta cero el criterio no permite decidir, puesto que pueden ocurrir las tres posibilidades, máximo o mínimo o punto de inflexión, basta tomar las funciones $f(x)=x^{4}, f(x)=-x^{4}, f(x)=x^{5}$.

Pareciera que Jazmín no queda conforme con su primera conclusión y procede a graficar la función $\mathrm{h}(\mathrm{x})=\mathrm{x}^{3}$, correctamente y mediante una flecha lo conecta con un texto, el cual contiene referencias a la segunda derivada que no son pertinentes, no obstante aquí en el gráfico visualiza el crecimiento de la función, pero no concluye.

Jazmín anota los criterios de la primera y segunda derivada para el cálculo de máximos y mínimos no obstante no logra concluir desde el criterio. Hace intervenir el registro gráfico, dibuja la curva de la función $h(x)=x^{3}$, pero no lo conecta con los criterios lo que muestra una falta de articulación.

En esta respuesta están en juego los registros algebraico-simbólico, el gráfico y el registro auxiliar de tabla (esquema).

Figura 2 - Producción de Lys

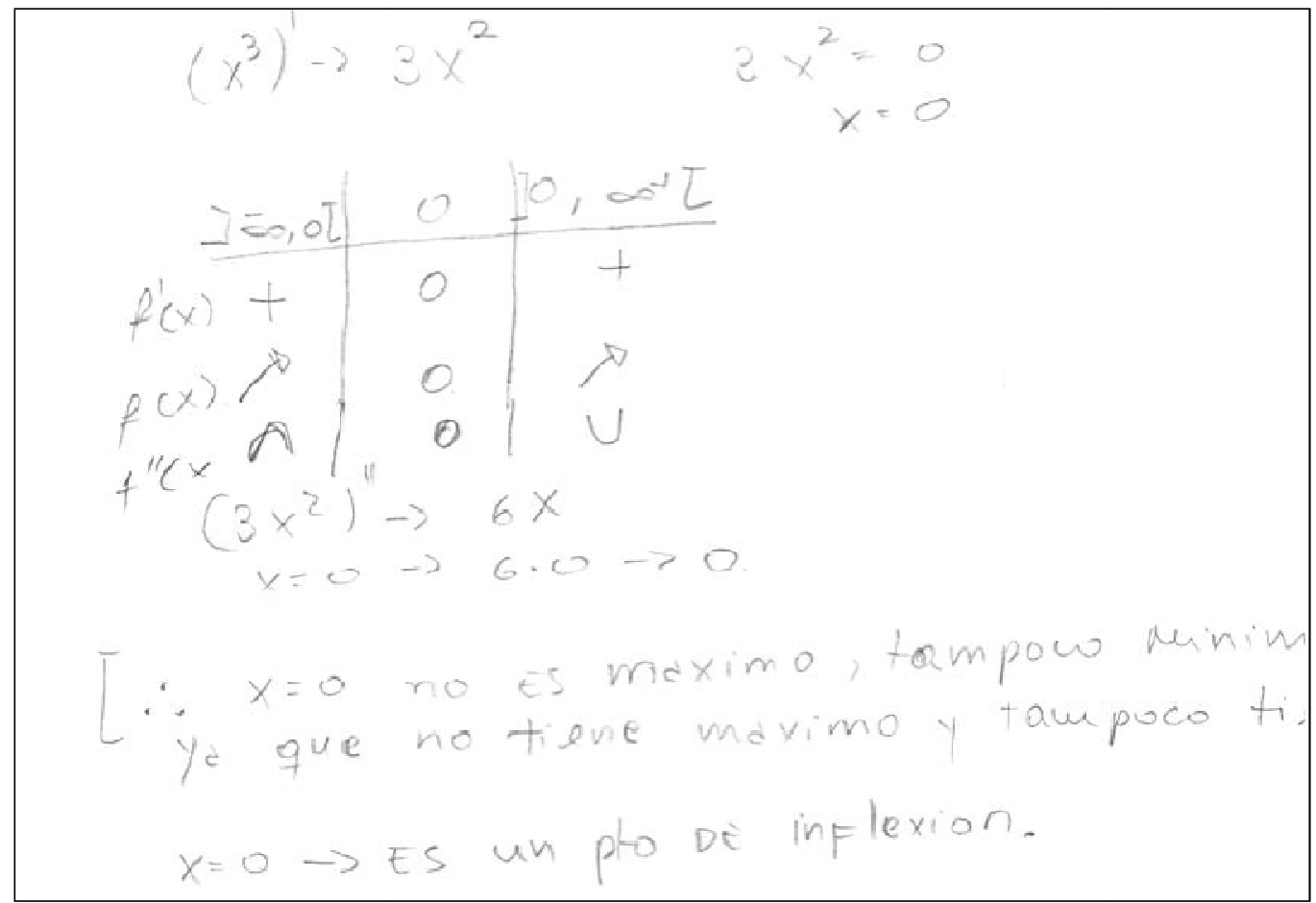

Lys, como en el caso anterior vemos que no pone atención al enunciado y realiza los cálculos para hallar puntos críticos y encuentra el $\mathrm{x}=0$ (dado en la hipótesis). 
Ella construye una tabla para el estudio de signos bien completa y correcta, en la primera línea aparecen los signos de f'(siendo h la función dada en el enunciado) en la segunda línea aparecen flechas, seguramente deducidas de los signos de la primera derivada.

Este desarrollo pone en juego los registros algebraico y de tabla; en la tabla tiene informaciones coherentes, pero que no coordina, pues de la segunda línea habría podido responder correctamente la pregunta, si hubiera relacionado las flechas con el crecimiento de la función h. Pero Lys, continúa con una tercera línea en su tabla, donde aparecen la concavidades, y el valor de la segunda derivada f' en cero.

Las informaciones obtenidas en esta línea también le habrían permitido responder correctamente la pregunta del problema, ya que tiene a la vista el cambio de concavidad. Ella infiere de aquí, que en $\mathrm{x}=0$ hay un punto de inflexión justificando así la respuesta que da en las frases finales de su desarrollo.

Figura 3 - Producción de Alelí

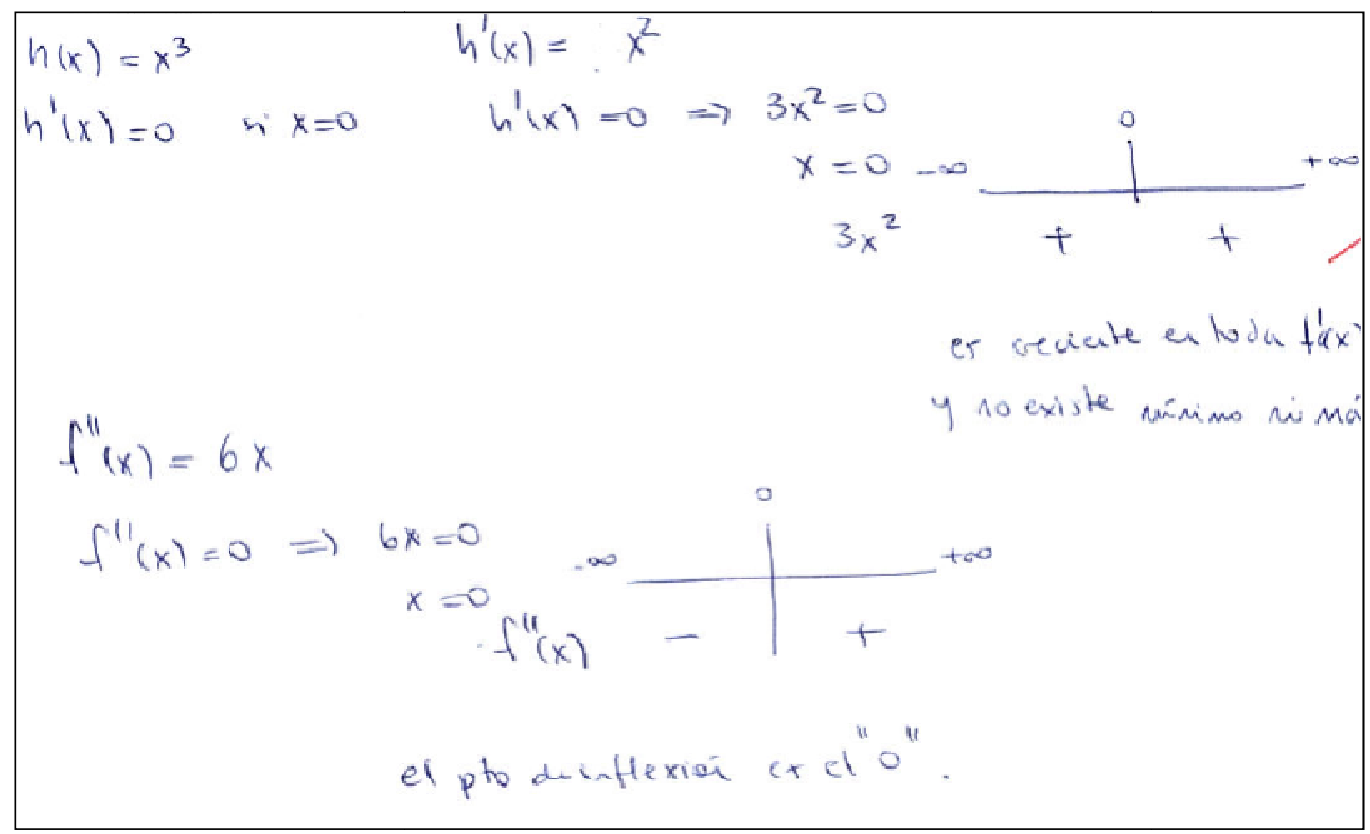

En este desarrollo vemos que ésta considera la hipótesis, luego el cálculo de la primera derivada de $\mathrm{h}$ y un esquema para el análisis de signos (de la primera derivada de h) de inmediato deduce que la función es creciente y concluye que no existe mínimo ni máximo. Por lo que tiene expresamente la respuesta al problema. 
Alelí ha puesto en juego el registro algebraico y el esquema de signos (registro auxiliar), los ha coordinado; no obstante ella no da por terminada su respuesta

El hecho que el enunciado del problema sea no rutinario y la falta de atención a la pregunta podría explicar la continuación del desarrollo calculando la segunda derivada de h que anota $\mathrm{f}$ " y el respectivo esquema de signos. Alelí concluye por el cambio de signos (que no los asocia expresamente con las concavidades de la curva) que cero es el punto de inflexión.

Alelí muestra que tiene conocimientos, los expone en forma coherente, pero no se da cuenta que en la primera parte ya ha respondido la pregunta del enunciado, ya que el problema no pide clasificar el punto, no obstante ella se impone esa tarea.

Este comportamiento estaría influenciado por la enseñanza, que usualmente aborda el problema de los puntos críticos, en forma típica: hallar los y clasificarlos. Y cuando la primera derivada es cero y no hay máximo ni mínimo entonces se insiste en que se tiene que recurrir a la segunda derivada.

Figura 4 - Producción de Fel

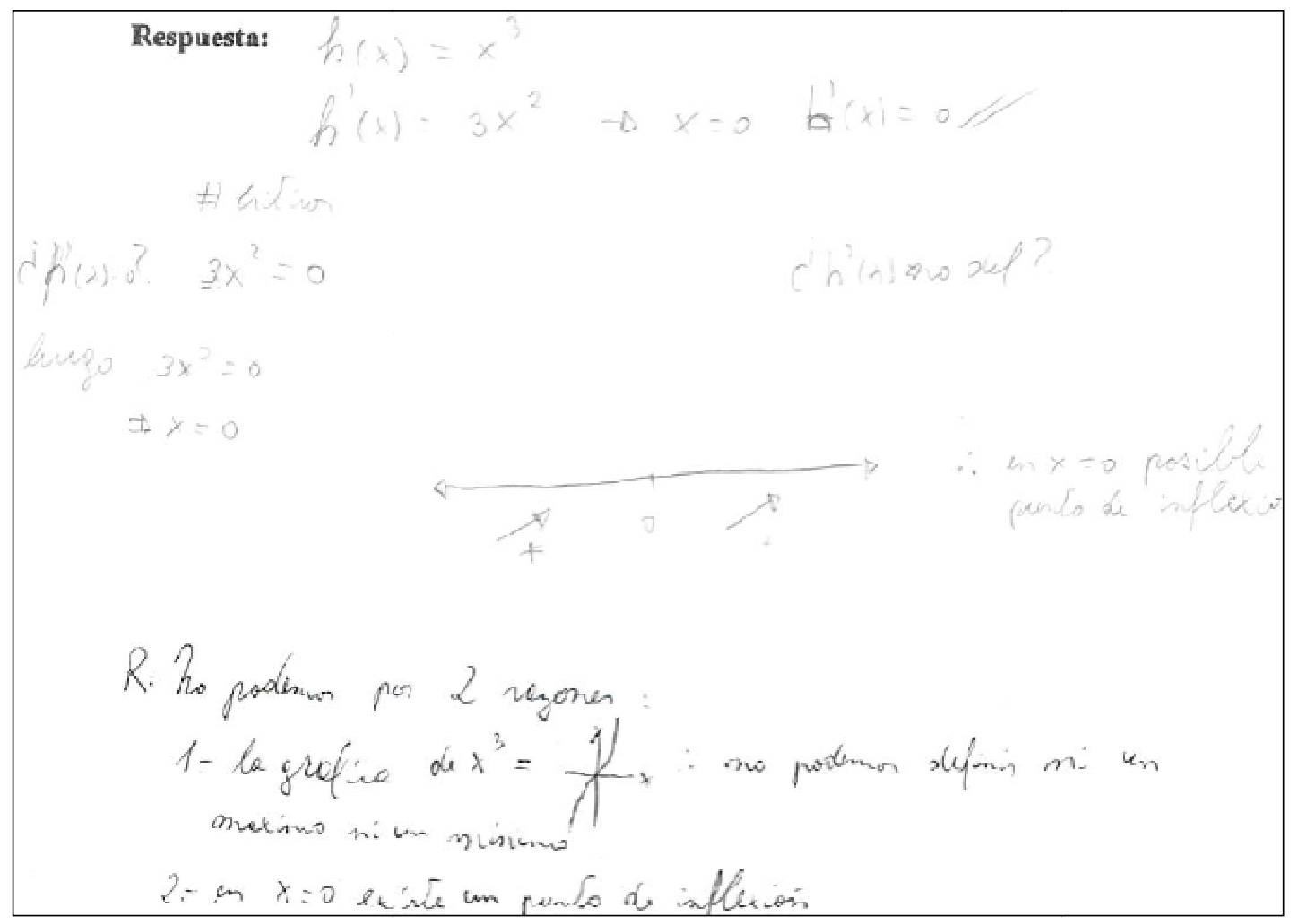


En la respuesta de Fel constatamos dos partes, en la primera no toma en cuenta el enunciado, calcula la primera derivada y reencuentra la hipótesis. A continuación construye el esquema de signos del cual podemos leer que la función es creciente, pero él no lo precisa y conjetura que en $\mathrm{x}=0$ "posible punto de inflexión", lo cual explicaría que dedujo implícitamente que no hay máximo ni mínimo, por ello supone posible punto de inflexión.

Fel pone en juego en esta primera parte el registro algebraico y el esquema de signos (registro auxiliar), los coordina conjeturando la posibilidad de un punto de inflexión.

En la segunda parte, responde directamente la pregunta del enunciado "No podemos asegurar".

Lo que justifica con la gráfica de la función $\mathrm{h}(\mathrm{x})=\mathrm{x}^{3}$, que suponemos conoce de antemano, así asegura que no hay máximo ni mínimo. Y su conjetura del posible punto de inflexión en $\mathrm{x}=0$ lo visualiza de la gráfica.

En esta segunda parte está en juego el registro gráfico y la interpretación visual.

Vemos que estas dos partes de la respuesta de Fel son independientes, y muestra que tiene conocimientos, pero no bien articulados. Ya que le hubiese sido suficiente cualquiera de las dos partes para responder correctamente la pregunta. Por otra parte es destacable que no recurriera a la segunda derivada como sus compañeros que ponen en juego un proceso mecánico de cálculos.

Figura 5 - Producción de Car

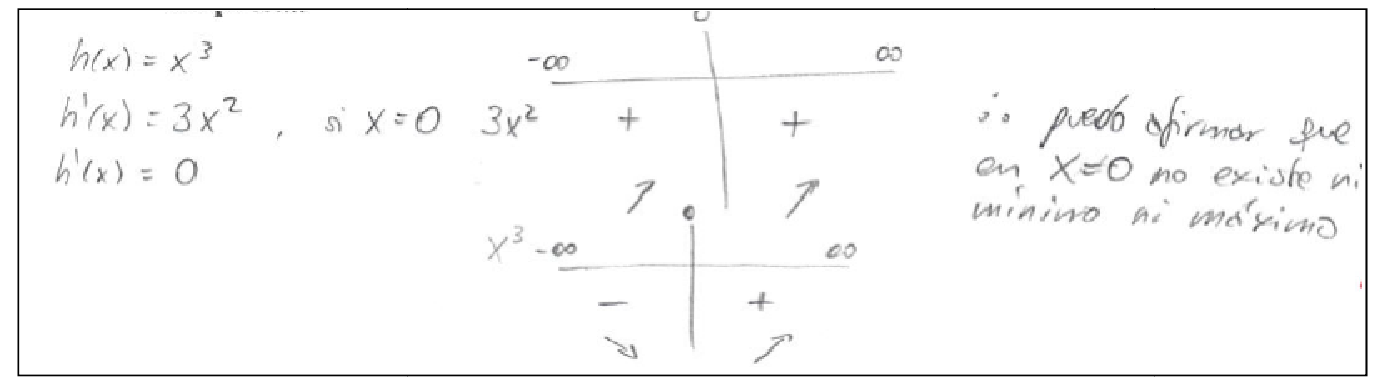

En este desarrollo vemos que calcula la primera derivada, encuentra la hipótesis y construye la tabla de signos, con datos implícitos. Si desde ella deduce su "texto a la derecha", habría respondido en forma aceptable; sin embargo sorprende la segunda tabla, la cual es de difícil interpretación, no se comprende el significado de las flechas, hay confusión ¿Relaciona signos con flechas?, ¿Flechas con monotonía de la función? 
La respuesta de Car muestra una debilidad en sus conocimientos, no se da cuenta que con su análisis de la primera tabla habría podido responder la pregunta.

Figura 6 - Producción de Goy

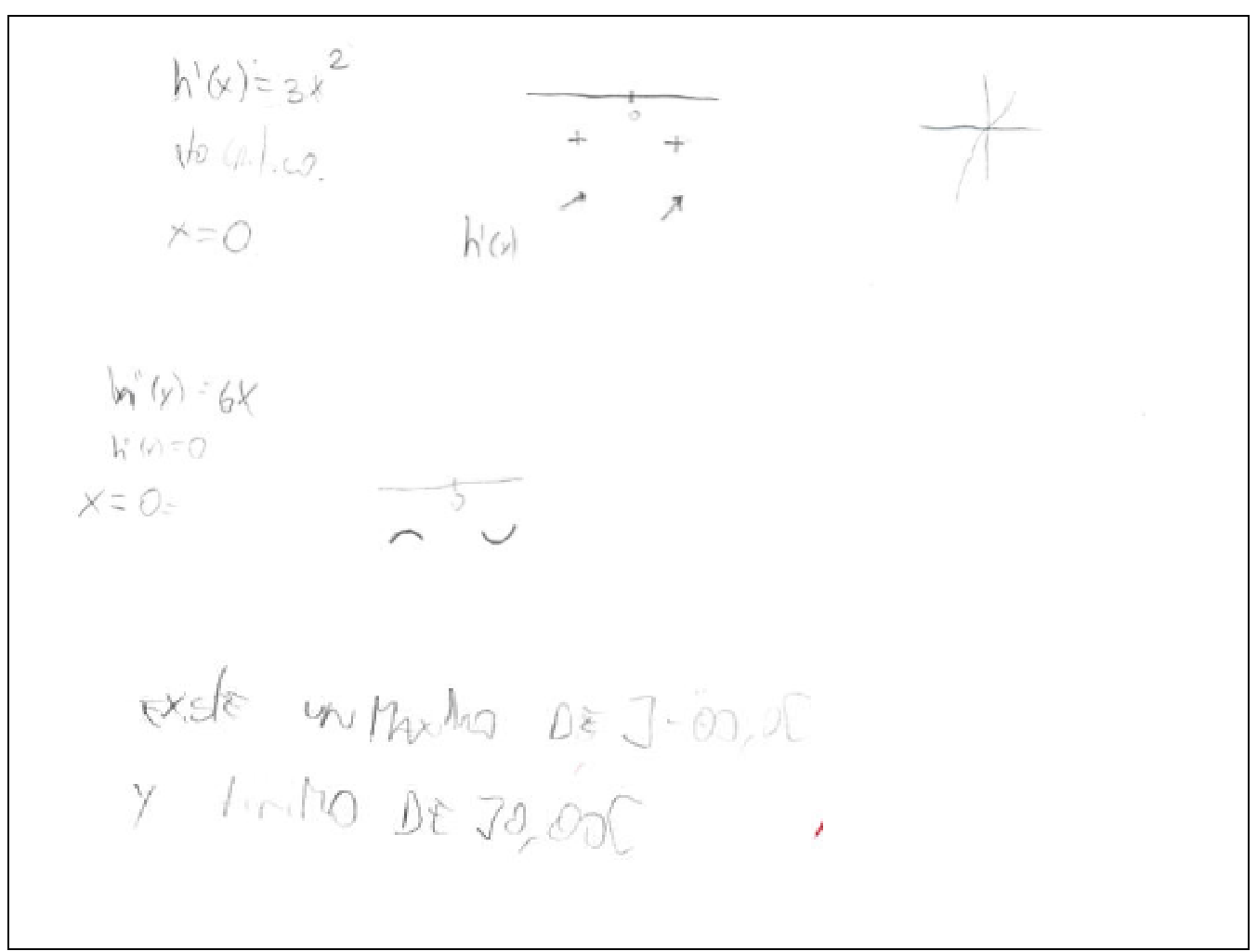

En esta respuesta Goy muestra conocimientos, que exhibe calculando la primera derivada, haciendo un esquema en que representa el dominio de la función mediante la recta real, destacando el 0 y colocando flechas que indican crecimiento de la función; dibuja a mano alzada el gráfico de la función. Con esto obtiene resultados que no interpreta, ya que con ellos hubiera podido responder a la pregunta.

Pero continúa derivando y estudia la concavidad mediante un esquema donde nuevamente se destaca la recta real.

Las concavidades que destaca del esquema, lo llevan a una conclusión muy particular, en este caso errónea, pero que perceptivamente las relaciona con los dibujos de los máximos y los mínimos.

Nuevamente en la respuesta de Goy notamos la falta de seguridad en los conocimientos, tal como Car no se da cuenta que en la primera parte de su desarrollo tiene elementos para responder correctamente. 


\section{Conclusiones}

El análisis de las respuestas ha permitido constatar dificultades de comprensión del enunciado ya que los estudiantes sin tomar en cuenta datos dados en el enunciado, operan aplicando técnicas operatorias para la determinación de máximos o mínimos.

En la mayoría de las respuestas podemos apreciar que los alumnos han aprendido un mecanismo para determinar máximos y mínimos, el cual se desencadena ante las palabras claves máximo o mínimo, sin tomar en cuenta lo que realmente el problema está solicitando y que no necesariamente se pide calcular. Ellos ponen en marcha el test de la primera derivada y continúan con el de la segunda, de inmediato sin darse cuenta de la necesidad de utilizar este segundo test.

Los registros que aparecen en la mayoría de las repuestas de los estudiantes, son el registro algebraico, el de tabla de signos (de doble entrada) o el registro auxiliar de esquema (simulando la recta real) y lenguaje natural. Estos registros habitualmente aparecen en textos y obras matemáticas, en particular en los de Cálculos Diferencial que con frecuencia constituyen la bibliografía de esta asignatura.

Los estudiantes muestran que han adquirido un procedimiento prácticamente algorítmico para responder este tipo de problemas, sin embargo el problema planteado al no ser rutinario, requería del estudiante una interpretación de las etapas del procedimiento en juego. En general la reflexión estuvo ausente, pues ellos hicieron cálculos sucesivos de la primera derivada para la monotonía y de la segunda derivada para la concavidad, sin darse cuenta que con la primera etapa de su trabajo tenían elementos de respuesta, si hubieran coordinado los registros en juego. Se nota que los estudiantes trabajan privilegiando el registro algebraico donde realizan sus tratamientos, sin reflexionar sobre los resultados que van obteniendo.

Trigueros M y Escandón C (2008) encontraron comportamientos similares con los estudiantes que ellos encuestaron, la mayoría de ellos calculan la primera y segunda derivada, privilegiando el criterio de la segunda derivada para responder a la pregunta planteada. Este mismo comportamiento que también se encontraron en Guzmán I. et al (2010). Esto debido a que operan siguiendo un procedimiento mecánico sin la apropiación de los objetos matemáticos en juego. 
Estos hallazgos, nos plantean algunas interrogantes, ¿esta similitud de comportamientos tiene relación con las formas de enseñanza en la universidad? ¿Qué concepción adquieren los estudiantes del Cálculo diferencial? ¿Lo perciben como una serie de algoritmos que hay que memorizar y poner en práctica y no como un cuerpo de conocimientos organizados?

Sugerimos repensar la pedagogía universitaria en el sentido de enfatizar sobre los objetos matemáticos integrados a un marco teórico con el fin de ir más allá de los algoritmos y los aprendizajes técnicas operatorias.

\section{Referencias bibliográficas}

DE LA TORRE, A. et al. El Método de de Máximos y Mínimos de Fermat. Revista de Educación Matemática, 002, p. 31-37, 2005.

DUVAL, R. Sémiosis et pensée humaine. Peter lang, Bern, 1995.

DUVAL, R. (Ed.). Conversion et articulation des représentations analogiques. Séminaire I.U.F.M. Nord Pas-de-Calais, 1, Villeneuve d'Ascq, 1999.

DUVAL, R. Décrire, visualiser, raisonner : quels «apprentissages premiers » de l'activité mathématique? Annales de Didactique et de Sciences cognitives, 8, p. 13-62, 2003a.

DUVAL, R. Langage, symboles, images, schemas... :de quelle manière interviennentils dans la compréhension, en mathématiques et en dehors des mathématiques? in Bolletino dei docenti di matematica.

DUVAL, R. La gaceta de la RSME, España, 9(1), p. 143-168, 2006.

GUZMÁN, I. et al. La apropiación de los criterios de optimización en Cálculo Diferencial, publicación on line: <http://sites.univ-lyon2.fr/asi5>, 2010.

GUZMÁN, I. Registros de representación, el aprendizaje de nociones relativas a funciones: voces de los estudiantes. Relime, n. 1. p. 5-21, 1998.

GUZMÁN, I. et al. ¿Cómo enseñan las derivadas los profesores de cálculo, en la Universidad? IV Encuentro internacional de Análisis Estadístico Implicativo Universitat Jaime I. España, 2007.

MORENO, S.; CUEVAS, C. Interpretaciones erróneas sobre los conceptos de máximos y mínimos en el cálculo diferencial. Revista Educación Matemática, 16(2), p. 93-104, 2004. 
TRIGUEROS, M.; ESCANDÓN, C. Los conceptos relevantes en el Aprendizaje de la Graficación. RMIE, 13(36), p. 59-85, 2008. 